\title{
XXVI. On magnetic Rays
}

\section{Louis T. More \& Edward G. Rieman M.A.}

To cite this article: Louis T. More \& Edward G. Rieman M.A. (1912) XXVI. On magnetic Rays, Philosophical Magazine Series 6, 24:140, 307-316, DOI: 10.1080/14786440808637332

To link to this article: http://dx.doi.org/10.1080/14786440808637332

册 Published online: 20 Apr 2009.

Submit your article to this journal 준

Џll Article views: 2

Q View related articles $₫$

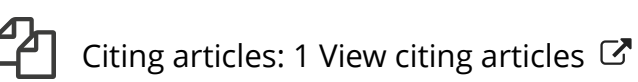


it will begin to diminish in accordance with the very rapid decrease of the contact-difference of aluminium and copper observed in ordinary cases. It is rather remarkable that, if the above theory be correct, the more electropositive a metal is the less the number of bound electrons in the atom, the effect of ultra-violet light should be to make a metal temporarily more electronegative, as one known effect is to expel electrons from the surface. It would seem as if it not only projected electrons out of the surface, but projected them into the atoms as well. It would be interesting to investigate whether, by charging the metal positively and thus preventing the expulsion of electrons, the decrease in contact-difference would also be prevented.

A rather striking experiment which, no doubt, is connected with the above subject is the following. If a plate of zinc or aluminium, or indeed of any metal-but the effect is more marked in the case of zinc and aluminium-be exposed to ultra-violet light for a short time, the light passing through a geometrically shaped aperture in a sereen, a distinet pattern will bo observed on the metal of the same shape as the aperture. It was observed in the case of copper, brass, silver, and platinum.

XXVI. On Magnetic Rays. By LouIs T. MoRe, Professor of Physics, and Edward F. Rivman, M.A., Hanna Fellow in Physics, Unirersity of Cincinnati*

DROFESSOR RIGHI has applied the term "magnetic rays" to certain phenomena which occur when an electric current in rarefied air is influenced by a strong magnetic field. It has, of course, been known for a long time that a magnetic field altered the path and appearance of such currents, but it was generally believed that all the effects noted were due to an orbital motion given to the cathode rays by the magnetic force. Professor Righi has investigated the subject most elaborately and has come to the conclusion that the action of the magnetic field may give rise also to a new and special kind of rays. His results have appeared in numerous articles $\dagger$, which have lately been made more accessible by being revised and collected in a book. He has not only given his own results but he has also prefaced them by an account of the work of his predecessors.

* Communicated by the Authors. 1910

† Mem. della $R$. Acegd. dell Ist. Bologna, 1s08 and 1909. Le Radium 
The brief abstract of the results and theory of Professor Righi which pertain to the subject of the present article is taken from a German translation of his book, published by Barth under the title Strahlende Materie und Magnetische Strahlen.

The apparatus best suited to show the magnetic rays consists of a Geissler tube about a metre long and five centimetres in diameter (fig. 1). The anode is either an

Fig. 1.

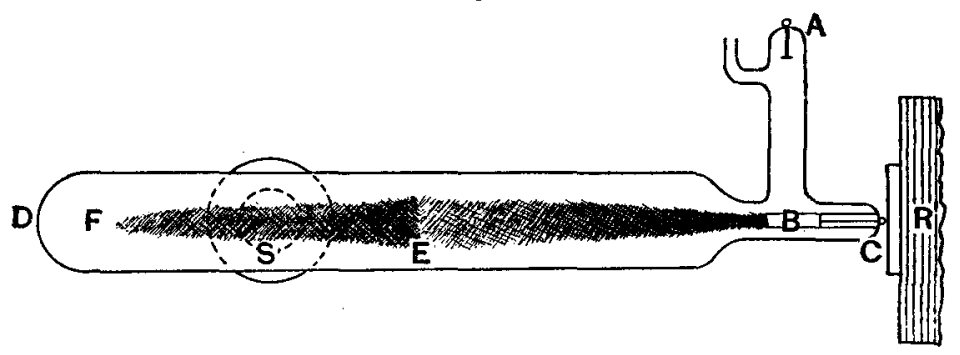

aluminium rod or disk placed in a branch tube, A. The cathode is situated at one end, C, in a somewhat narrower extension of the main tube ; it usually takes the form of a small aluminium disk, enclosed in a little glass tube that extends beyond the disk. The current is supplied by a high tension battery, an electrostatic machine, or an inductioncoil. $\mathbf{R}$ is a large electromagnet with the iron core removed, placed so that its axis coincides with the axis of the tube. The necessary field-strength varies from 500 to 2000 units at a distance of a centimetre and a half from the end of the spool, although most of the results are obtained near the lower limit.

When the field magnet, $R$, is not excited, the ordinary discharge takes place between the anode and cathode, and the tube BD remains dark; but if, while the discharge is passing, the magnet is excited to give a certain critical intensity of field depending on the size of the tube, the pressure of the gas, and the potential of the cathode, the whole tube is suddenly filled with a glow discharge, represented by the column BEF. The nature of this glow discharge can best be determined by an auxiliary magnet, $\mathrm{S}$, held near the tube and with its axis horizontal but perpendicular to that of the field magnet $R$.

While the magnetic field is weak, the two bluish-violet bright bands, separated by the negative dark spaces, are seen in the tube surrounding the cathode, and the pink anode 
column fills in the side tube. As the magnetic field increases the second negative bright band gradually lengthens and creeps into the large tube, until at the critical intensity the whole tube is filled suddenly with a glow discharge. If the magnetic field be still further increased, the discharge attains a maximum length and definition, and then diminishes in length. From $\mathrm{B}$ to $\mathrm{E}$ the light is similar in colour to the cathode rays in air, and from $\mathrm{E}$ to $\mathrm{F}$ it is reddish or more like a positive column. At $\mathrm{E}$, the two parts of the column are separated by a rather dark space.

If the magnet $\mathrm{S}$ is held with its axis horizontal and with its pole near the portion of the tube between $B$ and $E$, the rays bend in the arc of a horizontal curve. They bend toward the pole of the magnet, if this pole is of opposite sign to the nearer pole of the magnet $R$; and they bend directly away from $S$ if the poles are similar. On the other hand, an electrical field produces no effect on these rays. That is, the rays act as if thoy were composed of a moving stream of electrically neatral particles which are at the same time elementary magnets. Because of these actious, Professor Rigbi has applied the term "magnetic rays" to this part of the colunin.

The rest of the column EF has its origin in these magnetic rays and will be called the induced ray. If $S$ is held horizontally and with one pole near the induced ray, the column bends vertically like the positive column in a Geissler tube, The curvature of the ray is the same as would be produced by a similar magnetic field acting on a current of positively charged particles moving from $\mathrm{E}$ to $\mathrm{F}$. The action is seen more clearly if electrostatic discharges are prevented by enclosing the tube in an earthed metallic screen of copper gauze.

When the conditions are such that a very long induced column is formed, a different effect is shown by the magnet $S$. As it is moved along the tube a point is reached, indicated by $p$ in fig. 2, where the light splits into two helically

Fig. 2.

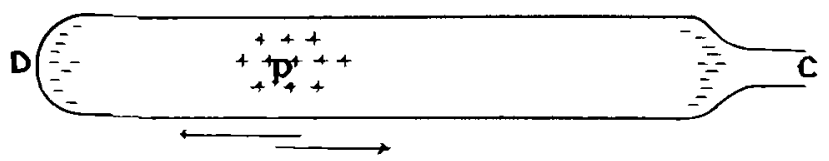

curved kands. Then as the magnet is moved toward $E$ or toward $F$, the bending of the column is opposite in curvature; if it bends up on one side, it bends down on the other. 
The direction in all cases is as if there were a virtual anode at $p$, due to a piling up of positively charged ions, and a positive current flowing toward each of the two ends, D and $\mathrm{C}$, as shown by the arrows. The discharge can also be shown to be intermittent, either by viewing it in a rotating mirror or by the fact that when it is intense it gives out a high-pitched musical note.

Professor Righi explains all these actions by assuming the magnetic rays to be due to what he calls planetary doublets (Doppelsterne) which consist each of a gas or metal ion, positively charged, and an electron revolving about it in an orbit. The bond of electrical attraction between them, due to their distauce apart, is of such a magnitude that, they may be classed as intermediate between a neutral atom and an atom entirely dissociated into an ion and an electron. He supposes that in a Geissler tube, and especially near the region of the second cathode bright space where there are many positive ions and electrons moving in opposite directions, neutral systems of particles exist in addition to the free, dissociated ions and electrons. These systems are of two kinds : neutral atoms which are formed when an electron with the proper speed comes so close to an ion as to be captured by it and held in a permanent chemical bond, and doublets where the speed of an electron is such that, while it is captured by the ion, they do not coalesce. The result in the second case is that the electron revolves about the ion as a satellite and forms an electrically neutral system, which is not very stable. Ordinarily these doublets are comparatively few in number and so short lived as not to be detected. Such a doublet, while it persists, should act like a magnetic shell, since the motion of the electron makes it equivalent to a small closed electric current.

As the presence of a magnetic field causes these doublets to persist, it must be shown that the magnetic force exerts a mechanical force on the system tending to increase its stability. The simple case of an electron rotating about a positive ion in a plane perpendicular to a uniform magnetic field has been discussed by Professor Righi, and he has shown that there is a resultant electromagnetic force on the electron in the plane of rotation and radial : either towards or away from the centre, depending on the direction of rotation of the electron. Consequently, all those doublets having a rotatory motion in one sense will have their stability increased by a given uniform magnetic tield, and those whose motion is in the opposite sense will be destroyed. If, however, the magnetic field be not uniform, then the 
resulting electromagnetic force is not in the plane of rotation but has a small translational component along the axis of rotation. The direction of this component is such that a doublet whose stability is increased by the magnetic field moves toward regions of lower magnetic intensity.

This hypothesis, if accepted, would account for the extension of the luminous column from the region near the cathode out into the large tube, since the stability of the doublets is increased and a translational motion given to them by the non-uniform field created by the magnet $\mathrm{R}$. It also explains why this column bends towards the magnet S or away from it according as the unlike or like poles of the two magnets are nearest each other. As these doublets move out into the tube, collisions with the gas in the tube, together with the diminishing electromagnetic force, cause their destruction, which is marked by the position $\mathrm{E}$. The freed electrons cause dissociation in the gas, and the limit of range of the positive ions is marked by the region $p$ which, on account of the accumulation of positive electricity there, acts like a virtual anode. The free electrons tend to go past the point $p$ and, in fact, usually travel to the far end of the tube for two reasons: the range of electrons is in general greater than that of positive ions, and the cathode exercises a repulsive force on the electron and an attractive force on the positive ion. The effect of this action is a Geissler tube discharge in the ionized gas toward both ends of the tube from this virtual anode. The oppositely directed currents in the induced column are indicated by the reversed curvature of the rays around the pole of $\mathbf{S}$ on the two sides of $p$.

Such, in brief, is Professor Righi's hypothesis to account for the action of a magnetic field on the current in a vacuum tube. It is simple, plausible, and accounts for most of the observed phenomena. Still there are some facts brought out in our experiments which indicate that the actions occurring are rather more complex and obscure. Apparently, Professor Righi limited his work to experiments with air; we find that other gases give rise to quite distinctive effects.

Our apparatus was, for the most part, similar to that of Professor Righi. Some of the tubes were obtained from Herrn Richard Miiller-Uri and were made according to Professor Righi's specifications; others were made in our laboratory. They were of various sizes; in some the anode was placed in a side tube, and in others the narrow portion of the tube was made long and pushed into the core of the field magnet. The anode was, in the latter tubes, inside the 
magnet and the cathode just in front of the pole ; the magnetic rays then proceeded from the back of the cathode. The current was obtained from an induction-coil. The magnet-coil was a large one, and the space in the core left by the tube was filled with soft-iron wires to increase the fieldstrength. The magnet was capable of developing a field much in excess of the requirements, since with a maximum current it became so intense as to destroy the rays; an effect noted by Professor Righi.

The tube was filled with air which had been thoroughly dried by passing it through sulphuric acid and phosphorus pentoxide. The first observations were made when the rays were not subjected to a magnetic field. As the pressure was reduced, the appearance of the current passed through the usual stages of the Geissler tube discharge. When a pressure of $0 \cdot 2 \mathrm{~mm}$. was reached, the two layers of negative glow and the cathode dark spaces were well defined in the little glass tube surrounding the cathode. Under such conditions Professor Righi observed that the large part of the tube remained dark, but our experience was that almost the whole tube was filled with pale bluish light. This discharge was evidently a mixture of cathode rays and an induced electrostatic discharge from the walls of the tube. The tube was not covered with a conducting earthed screen, and a finger touched to the glass produced a characteristic brush discharge. The electrostatic effect could be entirely eliminated by slipping over the tube an earthed screen of copper gauze, but even under those conditions some glow persisted in the tube. The trial was then made with the field magnet excited. As soon as the discharge was influenced by the magnetic field, the diffuse light in the large tube began to condense and contract into a column of light along the axis of the tube. Suddenly, when a critical pressure and magnetic field were reached, the appearance completely changed ; the induced electrostatic discharge at the far end of the tube disappeared, and in the centre of a faint and diffuse cathode discharge the true magnetic rays appeared as a sharply defined cone of blue light stretching along the axis of the tube and extending into the large part of the tube about one-fourth its length. These magnetic rays bent toward an auxiliary magnet if the poles of it and the field magnet nearest each other were opposite in sign, and were repelled if the poles were alike. At the end of the magnetic rays appeared the induced column EF (fig. 1). This light was rather reddish in colour, and showed the characteristic opposite curvature when the auxiliary magnet 
was moved along it, as indicated in fig. 2. Many observations were made of these phenomena, and both the pressure and magnetic field were varied so as to get all possible combinations. In general, the rays were more sensitive to changes of pressure than to variations in the magnetic intensity. For a given pressure, the magnetic field could be changed within wide limits without any other apparent effect than to increase or diminish somewhat the intensity and length of the rays; but with a given field, changes of pressure would soon destroy the rays altogether. In our apparatus the magnetic rays appeared quite suddenly at what may be called the critical pressure. This pressure was. approximately $0.03 \mathrm{~cm}$. As the current of the maynet was increased the rays gradually grew longer and having attained a maximum began to shorten until, with a sufficiently intense field, they suddenly ceased. The lowest pressure in which the rays could exist was about $0.006 \mathrm{~cm}$.; and, in general, as the pressure decreases the strength of the field must be increased.

The tube was next filled with illuminating gas, thoroughly dried. This gas is a natural gas obtained from wells and of unknown but probably complex composition. When the electric discharge was observed without a magnetic field, the cathode rays were less dense and of a paler blue than in air. The action of the magnetic field showed a decided difference. The magnetic field, apparently, was unable to contract the diffuse cathode rays into a well defined magnetic ray column. Even when a field was used sufficiently intense to destroy the action in air, the diffuse light filled nearly half the tube; the axial portion was slightly brighter, somewhat more blue in colour and extended only a short distance into the large tube, but no well defined column could be observed. This inner core showed the characteristic deflexions of magnetic rays when an auxiliary magnet was used. But the induced column of light at the end of the magnetic ray was lacking and could not be produced with any pressure and field. That the gas used in the tube should have such an influence on the nature of the magnetic rays is most difficult to explain by Professor Righi's hypothesis.

Various simple gases were used successively. Hydrogen, oxygen, nitrogen, and carbon-dioxide gases were prepared in as pure a state as possible and were thoroughly dried before being admitted to the tube, and the tube was filled and emptied many times to get rid of other gases, especially air. In no case, with any of these simple gases, could the discharge characteristic in air be obtained. The pressure 
and magnetic field were varied between wide ranges, from the limit where a simple glow discharge, without negative dark spaces, passed between the anode and cathode, to so great a vacuum that the induction-coil could not produce a luminous discharge at all. The appearance in each of the gases most nearly approaching that in air was a more or less diffuse cathode glow filling about half the large part of the tube with a core of magnetic rays short and sharply conical in shape, lying along its axis. The induced column at the end of these rays with a virtual anode and a reversed curvature was never obtaincd. If any of these gases were mixed with air, either by admitting a little air into the tube or by not removing all of it, then all the phenomena characteristic of the discharge in air were readily obtained. This fact made it certain that the gases were pure when the phenomena were not obtained.

Trials were then made with mixtures of the gases. Neither hydrogen or oxygen mixed with carbon dioxide in any proportion gave the effect. The magnetic rays were somewhat longer and larger, and there appeared occasionally a small induced column, but it always bent, in an auxiliary magnetic field, in the same direction throughout all its length, and the curvature indicated a positive current from the end of the magnetic ray column to the far end of the tube. There was no virtual anode in it with the positive current discharging in both directions as shown in fig. 2 .

Mixture of nitrogen and carbon dioxide.-This mixture gave all the effects of air ; both the magnetic rays and the induced column with a virtual anode were observed at the same pressures as for air. The discharge was rather less intense, but the magnetic field was able to condense all the diffuse glow into an axial column.

Mixture of nitrogen and oxygen.-The appearance was exactly that of air.

Mixture of nitrogen and hydrogen.-This mixture acted like air but much less certainly. The best proportion of gases was about six parts of nitrogen to one of hydrogen, If the proportionate part of nitrogen was much less, the induced column could not be produced.

We have been able to repeat satisfactorily the most important phenomena discovered hy Professor Righi when a magnetic field acts on the cathode rays in a tuhe containing air ; and we have, in addition, found that the nature of the gas has an important influence on this action. While it is not possible to state fully and exactly what these differences are and what their cause may be, yet our observations point 
to the conclusion that the magnetic rays are much more easily produced in air and artificial mixtures of nitrogen and oxygen than in the other mixtures we tried; and they, in turn, are more sensitive to the influence of the field than simple gasos, including the chemical compound carbon dioxide.

As regards what has been called the induced column, that is, the glow proceeding from the end of the magnetic rays which acts as if it were a current of charged ions moving in opposite directions from a virtual anode situated at a point in the column, we could obtain it in a well developed form only for air. While we also observed it with other mixtures containing nitrogen, the characteristic phenomena were less certain ; in other mixtures and in simple gases it was absent. 'Ihis seems to be correct, because if either pure nitrogen or oxygen were in the tube and a little of the other gas introduced, the induced column immediately declared itself.

These results are difficult to account for by Professor Righi's hypothesis. If the magnetic rays consist of a stream of doublets, each composed of a centre of a positively charged gas-ion, or metal-ion detached from the cathode, why should the doublets form more readily in a medium containing gasions of one gas rather than another, and especially when two gases not chemically combined are present.

Again, Professor Righi supposes that those doublets, which are made more stable by the action of a magnetic field and are driven by it toward regions of less intensity, have their stability decreased as their distance from the magnet increases. These doublets are finally destroyed by collisions, and their dissociated parts move on and ionize the gas. The range of these particles is shown by an excess of free positive electricity at a certain place in the tube which he calls a virtual anode. It is difficult to see why the existence of this virtual anode should depend on the gas. It might be claimed that for a light ion like hydrogen the virtual anode would be too near one end of the column, and for a heavier ion like nitrogen or oxygen too near the other end to make the reversed bending on the two sides of this anode evident. But this can hardly be the explanation because, while the column should then curve as a whole in one direction, yet the directions should be opposite for the columns of the two gases. This is not the case, for it always curved the same way for all gases and mixtures when the column was present, and the virtual anode could not be located.

Lastly, the hypothesis requires that a doublet be driven from an intense to a weaker part of the field, and this is in 
agreement with the fact that the magnetic ray curves toward an auxiliary magnet when the two nearest poles of it and che field magnet are unlike in sign, and is repelled when these poles are of like sign. Now, the magnetic field in these experiments is produced by a magnet placed at one end of the tube and the magnetic lines of force diverge in the tube. The fall of magnetic potential is also least rapid along the lines which lie near the axis of the tube. It would seem as if the magnetic rays, when the auxiliary magnet is not used, should be a diverging cone of light. Yet the most noticeable action of the magnetic field is to condense the light near the axis of the tube. And the stronger the field is made, the more sharply converging the cone of light becomes and the more contracted to the axial part of the tube.

These experiments should not be regarded as other than very incomplete, since the phenomena appear to be quite complicated. The attempt is being made to increase the discharge without unduly increasing the potential and to study the action of other gases and the state of the gas in different parts of the tube.

University of Cincinnati, April, 1912.

XXVII. Preliminary Note on the Electron Atmospheres of Metals. By R. W. Wood, Professor of Experimental Physics, Johns Hopkins University, and Adams Research Fellow of Columbia University *

TTHERE has been a good deal of controversy recently as to whether air at atmospheric pressure will carry a current of electricity, when the potential between the electrodes is less than what is known as the critical potential (something over 300 volts). Earhart, in a paper published in 1901 (Phil. Mag. [6] i. p. 147), described experiments which appeared to show that a discharge might occur with as low a potential as 32 volts, the gap between the electrodes being of the order of magnitude of the wave-length of light. This result was called into question by Almy, who worked with very small platinum beads as electrodes, and failed to obtain any evidence of discharge at voltages below 300. During the progress of the work about to be described a paper appeared in the Phil. Mag. by Anderson and Morrison (May 1912), which gives further evidence that currents may flow across.

* Communicated by the Author. 